\title{
About Aczél Inequality and Some Bounds for Several Statistical Indicators
}

\author{
Augusta Raţiu ${ }^{1, *,+} \mathbb{D}$ and Nicuşor Minculete ${ }^{2,+}$ \\ 1 Department of Mathematics and Informatics, Lucian Blaga University of Sibiu, Str. Dr. I. Raţiu, No. 5-7, \\ RO-550012 Sibiu, Romania \\ 2 Department of Mathematics and Computer Sciences, Transilvania University of Braşov, \\ 500091 Braşov, Romania; minculeten@yahoo.com \\ * Correspondence: augu2003@yahoo.com
}

Received: 22 March 2020; Accepted: 7 April 2020; Published: 13 April 2020

check for updates

\begin{abstract}
In this paper, we will study a refinement of the Cauchy-Buniakowski-Schwarz inequality and a refinement of the Aczél inequality by the technique of the monotony of a sequence. In the final part, we present some properties of bounds of several statistical indicators of variation.
\end{abstract}

Keywords: Cauchy-Buniakowski-Schwarz inequality; statistical indicators

\section{Introduction}

Many mathematicians have studied in their papers the inequality Cauchy-Buniakowski-Schwarz and Aczél's inequality (see [1-3]). We find the first inequality in numerical and complex analysis, Hilbert spaces theory, probability and statistics and it is defined as follows:

Let $\left(a_{1}, a_{2}, \ldots, a_{n}\right)$ and $\left(b_{1}, b_{2}, \ldots, b_{n}\right)$ be two sequences of real numbers, then

$$
\left(\sum_{i=1}^{n} a_{i}^{2}\right) \cdot\left(\sum_{i=1}^{n} b_{i}^{2}\right) \geq\left(\sum_{i=1}^{n} a_{i} b_{i}\right)^{2},
$$

with equality if and only if the sequences $\left(a_{1}, a_{2}, \ldots, a_{n}\right)$ and $\left(b_{1}, b_{2}, \ldots, b_{n}\right)$ are proportional.

Mărghidanu, Díaz-Barrero and Rădulescu in [4], proved a refinement of the inequality Cauchy-Buniakowski-Schwarz, given by the following: for arbitrary real sequences $\mathbf{a}=\left(a_{1}, a_{2}, \ldots, a_{n}\right)$ and $\mathbf{b}=\left(b_{1}, b_{2}, \ldots, b_{n}\right), b_{i} \neq 0, i=\overline{1, n}$, we have:

$$
\sum_{i=1}^{n} a_{i}^{2} \cdot \sum_{i=1}^{n} b_{i}^{2}-\left(\sum_{i=1}^{n} a_{i} b_{i}\right)^{2} \geq \max _{i, j \in\{1, \ldots, n\}} \frac{\left(a_{i} b_{j}-a_{j} b_{i}\right)^{2}}{b_{i}^{2}+b_{j}^{2}} \cdot \sum_{i=1}^{n} b_{i}^{2},
$$

for any $n \geq 2$, with equality if and only if the sequences $\mathbf{a}$ and $\mathbf{b}$ are proportional. In [5], Pop showed an improvement of inequality (2).

Two consequences can be obtained from above inequality, namely: for arbitrary sequence $\mathbf{a}=\left(a_{1}, a_{2}, \ldots, a_{n}\right), n \geq 2$, we have:

$$
n \sum_{i=1}^{n} a_{i}^{2}-\left(\sum_{i=1}^{n} a_{i}\right)^{2} \geq n \cdot \max _{i, j \in\{1, \ldots, n\}}\left(a_{i}-a_{j}\right)^{2}
$$

and

$$
n \sum_{i=1}^{n} a_{i}^{2}-\left(\sum_{i=1}^{n} a_{i}\right)^{2} \geq \max _{i, j \in\{1, \ldots, n\}} \frac{\left(a_{i}-a_{j}\right)^{2}}{a_{i}^{2}+a_{j}^{2}} \sum_{i=1}^{n} a_{i}^{2}
$$


with $a_{i} \neq 0, i=\overline{1, n}$.

In 1956, Aczél studied in [6], several methods in the theory of functional equations in one variable and showed the following inequality:

Let $\mathbf{a}=\left(a_{1}, a_{2}, \ldots, a_{n}\right)$ and $\mathbf{b}=\left(b_{1}, b_{2}, \ldots, b_{n}\right)$ be two sequences of positive real numbers such that:

$$
A^{2}-a_{1}^{2}-\ldots-a_{n}^{2}>0 \text { and } B^{2}-b_{1}^{2}-\ldots-b_{n}^{2}>0,
$$

where $A$ and $B$ are positive real numbers. Then

$$
\left(A^{2}-a_{1}^{2}-\ldots-a_{n}^{2}\right)\left(B^{2}-b_{1}^{2}-\ldots-b_{n}^{2}\right) \leq\left(A B-a_{1} b_{1}-\ldots-a_{n} b_{n}\right)^{2},
$$

with equality if and only if the sequences $\mathbf{a}$ and $\mathbf{b}$ are proportional, known as Aczél's inequality. This has many applications in the theory of functional equations in non-Euclidean geometry. Popoviciu [7], presented a generalized form of the inequality of Aczél:

Let $p, q>1$ be such that $\frac{1}{p}+\frac{1}{q}=1$ and $\mathbf{a}=\left(a_{1}, a_{2}, \ldots, a_{n}\right), \mathbf{b}=\left(b_{1}, b_{2}, \ldots, b_{n}\right)$ be two sequences of positive real numbers such that:

$$
A^{p}-a_{1}^{p}-\ldots-a_{n}^{p}>0 \text { and } B^{q}-b_{1}^{q}-\ldots-b_{n}^{q}>0,
$$

where $A$ and $B$ are positive real numbers. Then

$$
\left(A^{p}-a_{1}^{p}-\ldots-a_{n}^{p}\right)^{1 / p}\left(B^{q}-b_{1}^{q}-\ldots-b_{n}^{q}\right)^{1 / q} \leq A B-a_{1} b_{1}-\ldots-a_{n} b_{n}
$$

with equality if and only if the sequences $\mathbf{a}$ and $\mathbf{b}$ are proportional.

In the special case $p=q=2$, we deduce the classical Aczél inequality.

In Section 2, we study a refinement of the Cauchy-Buniakowski-Schwarz inequality and a refinement of the Aczél inequality by the technique of the monotony of a sequence. In Section 3, there are presented new bounds for the statistical indicators related to variance, standard deviation and coefficient of variation.

\section{Refinements of the Cauchy-Buniakowski-Schwarz Inequality and of the Aczél Inequality}

Next, we present a refinement of Cauchy-Buniakowski-Schwarz inequality, thus:

Theorem 1. For arbitrary real sequences $\mathbf{a}=\left(a_{1}, a_{2}, \ldots, a_{n}\right)$ and $\mathbf{b}=\left(b_{1}, b_{2}, \ldots, b_{n}\right), \mathbf{a}, \mathbf{b} \neq \mathbf{0}$, we have:

$$
\sqrt{\sum_{i=1}^{n} a_{i}^{2} \cdot \sum_{i=1}^{n} b_{i}^{2}}-\sum_{i=1}^{n} a_{i} b_{i} \geq \frac{1}{2} \min \{A, B\} \sum_{i=1}^{n}\left(a_{i}-b_{i}\right)\left(\frac{a_{i}}{A}-\frac{b_{i}}{B}\right) \geq 0,
$$

where $A=\sqrt{\sum_{i=1}^{n} a_{i}^{2}}$ and $B=\sqrt{\sum_{i=1}^{n} b_{i}^{2}}$ with equality if and only if the sequences $\mathbf{a}$ and $\mathbf{b}$ are proportional.

Proof. We have the following:

$$
\begin{aligned}
& \sum_{i=1}^{n}\left(a_{i}-b_{i}\right)\left(\frac{a_{i}}{A}-\frac{b_{i}}{B}\right)=\sum_{i=1}^{n}\left(\frac{a_{i}^{2}}{A}-\frac{a_{i} b_{i}}{B}-\frac{a_{i} b_{i}}{A}+\frac{b_{i}^{2}}{B}\right) \\
= & A+B-\frac{A+B}{A B} \sum_{i=1}^{n} a_{i} b_{i}=\frac{A+B}{A B}\left(A B-\sum_{i=1}^{n} a_{i} b_{i}\right) \geq 0 .
\end{aligned}
$$

Therefore, we obtain:

$$
A B-\sum_{i=1}^{n} a_{i} b_{i}=\frac{A B}{A+B} \sum_{i=1}^{n}\left(a_{i}-b_{i}\right)\left(\frac{a_{i}}{A}-\frac{b_{i}}{B}\right) .
$$


But, we have the inequality $\frac{A B}{A+B} \geq \frac{1}{2} \min \{A, B\}$, and using the equality (8), we deduce the inequality of the statement.

Corollary 1. For arbitrary sequence $\mathbf{a}=\left(a_{1}, a_{2}, \ldots, a_{n}\right), n \geq 2$, and $b>0$, we have:

$$
\sqrt{n \sum_{i=1}^{n} a_{i}^{2}}-\sum_{i=1}^{n} a_{i} \geq \frac{1}{2 A \sqrt{n}} \min \{A, b \sqrt{n}\} \sum_{i=1}^{n}\left(\frac{a_{i}}{b}-1\right)\left(a_{i} \sqrt{n}-A\right) \geq 0,
$$

where $A=\sqrt{\sum_{i=1}^{n} a_{i}^{2}}$.

Proof. If we take in inequality (7), $b_{1}=b_{2}=\ldots=b_{n}=b>0$, then we obtain:

$$
b \sqrt{n \sum_{i=1}^{n} a_{i}^{2}}-b \sum_{i=1}^{n} a_{i} \geq \frac{1}{2} \min \{A, b \sqrt{n}\} \sum_{i=1}^{n}\left(a_{i}-b\right)\left(\frac{a_{i}}{A}-\frac{b}{b \sqrt{n}}\right) \geq 0 .
$$

We divide with $b$ above relation and we obtain:

$$
\sqrt{n \sum_{i=1}^{n} a_{i}^{2}}-\sum_{i=1}^{n} a_{i} \geq \frac{1}{2} \min \{A, b \sqrt{n}\} \sum_{i=1}^{n}\left(\frac{a_{i}}{b}-1\right)\left(\frac{a_{i} \sqrt{n}-A}{A \sqrt{n}}\right) \geq 0,
$$

which implies the inequality of the statement.

Theorem 2. For arbitrary sequences of positive real numbers $\mathbf{a}=\left(a_{1}, a_{2}, \ldots, a_{n}\right)$ and $\mathbf{b}=\left(b_{1}, b_{2}, \ldots, b_{n}\right)$ such that:

$$
A^{2}-a_{1}^{2}-\ldots-a_{n}^{2}>0 \text { and } B^{2}-b_{1}^{2}-\ldots-b_{n}^{2}>0, n \geq 1,
$$

where $A$ and $B$ are positive real numbers, we have:

$$
\begin{aligned}
& \left(A B-\sum_{i=1}^{n} a_{i} b_{i}\right)^{2}-\left(A^{2}-\sum_{i=1}^{n} a_{i}^{2}\right)\left(B^{2}-\sum_{i=1}^{n} b_{i}^{2}\right) \\
& \geq \max _{i \in\{1, \ldots, n\}} \frac{\left(A b_{i}-B a_{i}\right)^{2}}{B^{2}-b_{i}^{2}}\left(B^{2}-\sum_{i=1}^{n} b_{i}^{2}\right) \geq 0,
\end{aligned}
$$

with equality if and only if the sequences $\mathbf{a}$ and $\mathbf{b}$ are proportional.

Proof. We use the technique of the monotony, as in [8] and [9], to the sequence $G_{n}(\mathbf{a}, \mathbf{b})$, which is defined as follows:

$$
G_{n}(\mathbf{a}, \mathbf{b})=\frac{\left(A B-\sum_{i=1}^{n} a_{i} b_{i}\right)^{2}}{B^{2}-\sum_{i=1}^{n} b_{i}^{2}}-A^{2}+\sum_{i=1}^{n} a_{i}^{2}, n \geq 1
$$

for $k \leq n$, we have:

$$
G_{k+1}(\mathbf{a}, \mathbf{b})-G_{k}(\mathbf{a}, \mathbf{b})=\frac{\left(A B-\sum_{i=1}^{k} a_{i} b_{i}-a_{k+1} b_{k+1}\right)^{2}}{B^{2}-\sum_{i=1}^{k} b_{i}^{2}-b_{k+1}^{2}}+a_{k+1}^{2}-\frac{\left(A B-\sum_{i=1}^{k} a_{i} b_{i}\right)^{2}}{B^{2}-\sum_{i=1}^{k} b_{i}^{2}}
$$


Using Bergström's inequality for two terms, we have:

$$
\frac{\left(A B-\sum_{i=1}^{k} a_{i} b_{i}-a_{k+1} b_{k+1}\right)^{2}}{B^{2}-\sum_{i=1}^{k} b_{i}^{2}-b_{k+1}^{2}}+\frac{\left(a_{k+1} b_{k+1}\right)^{2}}{b_{k+1}^{2}} \geq \frac{\left(A B-\sum_{i=1}^{k} a_{i} b_{i}\right)^{2}}{B^{2}-\sum_{i=1}^{k} b_{i}^{2}} .
$$

This means that $G_{k+1}(\mathbf{a}, \mathbf{b})-G_{k}(\mathbf{a}, \mathbf{b}) \geq 0$, so the sequence $G_{k}(\mathbf{a}, \mathbf{b})$ is increasing. Therefore, we obtain $G_{n}(\mathbf{a}, \mathbf{b}) \geq G_{n-1}(\mathbf{a}, \mathbf{b}) \geq \ldots \geq G_{2}(\mathbf{a}, \mathbf{b}) \geq G_{1}(\mathbf{a}, \mathbf{b})$. But,

$$
G_{1}(\mathbf{a}, \mathbf{b})=\frac{\left(A B-a_{1} b_{1}\right)^{2}}{B^{2}-b_{1}^{2}}-A^{2}+a_{1}^{2}=\frac{\left(A b_{1}-B a_{1}\right)^{2}}{B^{2}-b_{1}^{2}},
$$

and taking into account that we can rearrange the terms of the two sequences, we have the inequality:

$$
G_{n}(\mathbf{a}, \mathbf{b}) \geq \max _{i \in\{1, \ldots, n\}} \frac{\left(A b_{i}-B a_{i}\right)^{2}}{B^{2}-b_{i}^{2}}
$$

Multiplying by $B^{2}-\sum_{i=1}^{n} b_{i}^{2}>0$, we deduce the inequality of the statement.

Remark 1. In paper [10], F. Qi, P. Cerone, S. Dragomir and H.M. Srivastava have developed alternative proofs for the monotony.

Theorem 3. Let $p, q>1$ be such that $\frac{1}{p}+\frac{1}{q}=1$ and $\mathbf{a}=\left(a_{1}, a_{2}, \ldots, a_{n}\right), \mathbf{b}=\left(b_{1}, b_{2}, \ldots, b_{n}\right)$ be two sequences of positive real numbers such that:

$$
A^{p}-a_{1}^{p}-\ldots-a_{n}^{p}>0 \text { and } B^{q}-b_{1}^{q}-\ldots-b_{n}^{q}>0, n \geq 1,
$$

where $A$ and $B$ are positive real numbers. Then, we have:

$$
\begin{gathered}
A B-a_{1} b_{1}-\ldots-a_{n} b_{n}-\left(A^{p}-a_{1}^{p}-\ldots-a_{n}^{p}\right)^{1 / p}\left(B^{q}-b_{1}^{q}-\ldots-b_{n}^{q}\right)^{1 / q} \\
\geq \max _{i \in\{1, \ldots, n\}}\left\{A B-a_{i} b_{i}-\left(A^{p}-a_{i}^{p}\right)^{1 / p}\left(B^{q}-b_{i}^{q}\right)^{1 / q}\right\} \geq 0,
\end{gathered}
$$

with equality if and only if the sequences $\mathbf{a}$ and $\mathbf{b}$ are proportional.

Proof. We consider the sequence:

$$
H_{n}(\mathbf{a}, \mathbf{b})=A B-a_{1} b_{1}-\ldots-a_{n} b_{n}-\left(A^{p}-a_{1}^{p}-\ldots-a_{n}^{p}\right)^{1 / p}\left(B^{q}-b_{1}^{q}-\ldots-b_{n}^{q}\right)^{1 / q} .
$$

We study the monotony of the sequence $H_{k}(\mathbf{a}, \mathbf{b}), k \leq n$. Since

$$
\begin{gathered}
H_{k+1}(\mathbf{a}, \mathbf{b})-H_{k}(\mathbf{a}, \mathbf{b})=\left(A^{p}-a_{1}^{p}-\ldots-a_{k}^{p}\right)^{1 / p}\left(B^{q}-b_{1}^{q}-\ldots-b_{k}^{q}\right)^{1 / q} \\
-a_{k+1} b_{k+1}-\left(A^{p}-a_{1}^{p}-\ldots-a_{k+1}^{p}\right)^{1 / p}\left(B^{q}-b_{1}^{q}-\ldots-b_{k+1}^{q}\right)^{1 / q} .
\end{gathered}
$$

which involves,

$$
H_{k+1}(\mathbf{a}, \mathbf{b})-H_{k}(\mathbf{a}, \mathbf{b})=M N-a_{k+1} b_{k+1}-\left(M^{p}-a_{k+1}^{p}\right)^{1 / p}\left(N^{q}-b_{k+1}^{q}\right)^{1 / q},
$$


where we have $M^{p}=A^{p}-a_{1}^{p}-\ldots-a_{k}^{p}$ and $N^{q}=B^{q}-b_{1}^{q}-\ldots-b_{k}^{q}$. But using inequality (6) for two terms, we deduce:

$$
\left(M^{p}-a_{k+1}^{p}\right)^{1 / p}\left(N^{q}-b_{k+1}^{q}\right)^{1 / q} \leq M N-a_{k+1} b_{k+1}
$$

which involves the inequality $H_{n+1}(\mathbf{a}, \mathbf{b})-H_{n}(\mathbf{a}, \mathbf{b}) \geq 0$. Therefore the sequence $H_{n}$ is increasing, so, we obtain:

$$
\begin{gathered}
H_{n}(\mathbf{a}, \mathbf{b}) \geq H_{n-1}(\mathbf{a}, \mathbf{b}) \geq \ldots \geq H_{2}(\mathbf{a}, \mathbf{b}) \geq H_{1}(\mathbf{a}, \mathbf{b}) \\
=A B-a_{1} b_{1}-\left(A^{p}-a_{1}^{p}\right)^{1 / p}\left(B^{q}-b_{1}^{q}\right)^{1 / q} .
\end{gathered}
$$

Consequently, we deduce the statement.

Remark 2. In the particular case $p=q=2$, we deduce another refinement of the classical Aczél inequality, given by:

$$
\begin{gathered}
A B-a_{1} b_{1}-\ldots-a_{n} b_{n}-\left(A^{2}-a_{1}^{2}-\ldots-a_{n}^{2}\right)^{1 / 2}\left(B^{2}-b_{1}^{2}-\ldots-b_{n}^{2}\right)^{1 / 2} \\
\geq \max _{i \in\{1, \ldots, n\}}\left\{A B-a_{i} b_{i}-\left(A^{2}-a_{i}^{2}\right)^{1 / 2}\left(B^{2}-b_{i}^{2}\right)^{1 / 2}\right\} \geq 0,
\end{gathered}
$$

with equality if and only if the sequences $\mathbf{a}$ and $\mathbf{b}$ are proportional.

Extending Popoviciu's result to three sequences of positive real numbers, we obtain the following refinement:

Theorem 4. Let $p, q, r>1$ be such that $\frac{1}{p}+\frac{1}{q}+\frac{1}{r}=1$ and $\mathbf{a}=\left(a_{1}, a_{2}, \ldots, a_{n}\right), \mathbf{b}=\left(b_{1}, b_{2}, \ldots, b_{n}\right)$, $\mathbf{c}=\left(c_{1}, c_{2}, \ldots, c_{n}\right)$ three sequences of positive real numbers such that $A^{p}-a_{1}^{p}-\ldots-a_{n}^{p}>0$, $B^{q}-b_{1}^{q}-\ldots-b_{n}^{q}>0$, and $C^{r}-c_{1}^{r}-\ldots-c_{n}^{r}>0, n \geq 1$, where $A, B$ and $C$ are positive real numbers. Then, we have:

$$
\begin{gathered}
A B C-a_{1} b_{1} c_{1}-\ldots-a_{n} b_{n} c_{n}-\left(A^{p}-a_{1}^{p}-\ldots-a_{n}^{p}\right)^{1 / p}\left(B^{q}-b_{1}^{q}-\ldots-b_{n}^{q}\right)^{1 / q}\left(C^{r}-c_{1}^{r}-\ldots-c_{n}^{r}\right)^{1 / r} \\
\quad \geq \max _{i \in\{1, \ldots, n\}}\left\{A B C-a_{i} b_{i} c_{i}-\left((A B)^{\frac{r}{r-1}}-\left(a_{i} b_{i}\right)^{\frac{r}{r-1}}\right)^{\frac{r-1}{r}}\left(C^{r}-c_{i}^{r}\right)^{1 / r}\right\} \geq 0 .
\end{gathered}
$$

Proof. From hypothesis, we have $\frac{1}{p}+\frac{1}{q}+\frac{1}{r}=1$, which becomes $\frac{1}{p}+\frac{1}{q}=\frac{r-1}{r}$, so, we deduce $\frac{1}{\frac{p(r-1)}{r}}+\frac{1}{\frac{q(r-1)}{r}}=1$. Using Popoviciu's inequality for two sequences, we have:

$$
\begin{gathered}
{\left[\left(A^{p}-a_{1}^{p}-\ldots-a_{n}^{p}\right)^{1 / p}\left(B^{q}-b_{1}^{q}-\ldots-b_{n}^{q}\right)^{1 / q}\right]^{r /(r-1)}=} \\
\left(A^{p}-a_{1}^{p}-\ldots-a_{n}^{p}\right)^{r / p(r-1)}\left(B^{q}-b_{1}^{q}-\ldots-b_{n}^{q}\right)^{r / q(r-1)}= \\
{\left[\left(\left(A^{r /(r-1)}\right)^{p(r-1) / r}-\left(a_{1}^{r /(r-1)}\right)^{p(r-1) / r}-\ldots-\left(a_{n}^{r /(r-1)}\right)^{p(r-1) / r}\right)^{r / p(r-1)}\right]^{r / p(r-1)}} \\
{\left[\left(\left(B^{r /(r-1)}\right)^{q(r-1) / r}-\left(b_{1}^{r /(r-1)}\right)^{q(r-1) / r}-\ldots-\left(b_{n}^{r /(r-1)}\right)^{q(r-1) / r}\right)^{r / q(r-1)}\right]^{r / q(r-1)}} \\
\leq(A B)^{r /(r-1)}-\left(a_{1} b_{1}\right)^{r /(r-1)}-\ldots-\left(a_{n} b_{n}\right)^{r /(r-1)},
\end{gathered}
$$

which is equivalent to:

$$
\begin{aligned}
& \left(A^{p}-a_{1}^{p}-\ldots-a_{n}^{p}\right)^{1 / p}\left(B^{q}-b_{1}^{q}-\ldots-b_{n}^{q}\right)^{1 / q} \\
\leq & {\left[(A B)^{r /(r-1)}-\left(a_{1} b_{1}\right)^{r /(r-1)}-\ldots-\left(a_{n} b_{n}\right)^{r /(r-1)}\right]^{(r-1) / r} . }
\end{aligned}
$$


But, using above relation and Theorem 3, we have:

$$
\begin{gathered}
\left(A^{p}-a_{1}^{p}-\ldots-a_{n}^{p}\right)^{1 / p}\left(B^{q}-b_{1}^{q}-\ldots-b_{n}^{q}\right)^{1 / q}\left(C^{r}-c_{1}^{r}-\ldots-c_{n}^{r}\right)^{1 / r} \\
\leq\left[(A B)^{r /(r-1)}-\left(a_{1} b_{1}\right)^{r /(r-1)}-\ldots-\left(a_{n} b_{n}\right)^{r /(r-1)}\right]^{1-1 / r}\left(C^{r}-c_{1}^{r}-\ldots-c_{n}^{r}\right)^{1 / r} \\
\leq A B C-a_{1} b_{1} c_{1}-\ldots-a_{n} b_{n} c_{n}-\max _{i \in\{1, \ldots, n\}}\left\{A B C-a_{i} b_{i} c_{i}-\left((A B)^{\frac{r}{r-1}}-\left(a_{i} b_{i}\right)^{\frac{r}{r-1}}\right)^{\frac{r-1}{r}}\left(C^{r}-c_{i}^{r}\right)^{1 / r}\right\} .
\end{gathered}
$$

Consequently, we deduce the inequality of the statement.

\section{Applications to Several Statistical Indicators}

Analysis of social-economic phenomena with the statistical indicators help us to characterize the evolution of these phenomena, (see [11]). Therefore, below we present some of the statistical indicators that characterize a data series.

The mean of individual values of a data series is the expression level synthesis into a single representative of everything is essential, typical and objective in appearance, manifestation and its development.

The arithmetic mean $(\bar{X})$ is used when the phenomenon under investigation register changes approximately constant in arithmetic progression.

Let $X=\left(\begin{array}{l}x_{i} \\ p_{i}\end{array}\right)_{1 \leq i \leq n}$ be a discrete random variable with probabilities $P\left(X=x_{i}\right)=p_{i}=\frac{1}{n}$ for any $i=\overline{1, n}$, then expression of simple arithmetic mean is: $\bar{X}=\frac{x_{1}+x_{2}+\ldots+x_{n}}{n}=\frac{\sum_{i=1}^{n} x_{i}}{n}$.

The geometric mean $\left(\bar{X}_{g}\right)$ is that value which shows that if you replace each individual value, their product would not change and we have the formula: $\bar{X}_{g}=\sqrt[n]{x_{1} \cdot x_{2} \cdot \ldots \cdot x_{n}}$.

In statistics, by general notion of scattering (variance or dispersion) refer to the individual values of measurable deviations from the central value (typical).

Among the main indicators characterizing the scattering one can include: dispersion, standard deviation and coefficient of variation.

The dispersion (or variance) $(\operatorname{Var}(X))$ represents the simple arithmetic or weighted mean of individual deviations squared from their central tendency. is the parameter that characterizes the normal distribution and we have the formula: $\operatorname{Var}(X)=\frac{\left(x_{1}-\bar{X}\right)^{2}+\left(x_{2}-\bar{X}\right)^{2}+\ldots+\left(x_{n}-\bar{X}\right)^{2}}{n}$.

The standard deviation $\left(\sigma_{\bar{X}}\right)$ has a similar role with average linear deviation, but keeping the dispersion characteristics; statistics used this indicator which is calculated as mean of individual deviations squared from their central tendency, and the interval $\left(\bar{X}-\sigma_{\bar{X}} ; \bar{X}+\sigma_{\bar{X}}\right)$ is the medium interval of variation and we have the following formula: $\sigma_{\bar{X}}=\sqrt{\operatorname{Var}(X)}$.

The coefficient of variation $\left(C_{V}\right)$ is a relative measure of the scattering, which describes the percentage standard deviation from the arithmetic mean, $C_{V}=\frac{\sigma_{\bar{X}}}{\bar{X}}$. The covariance is a measure of how much two random variables $X$ and $Y$ change together at the same time and is defined as $\operatorname{Cov}(X, Y)=E[(X-E[X])(Y-E[Y])]$, and is equivalent to the form $\operatorname{Cov}(X, Y)=E[X Y]-E[X][Y]$. we find the inequality of Cauchy-Schwarz for discrete random variables given by: $|\operatorname{Cov}(X, Y)| \leq$ $\sqrt{\operatorname{Var}(X) \operatorname{Var}(Y)}$.

In 1935, Popoviciu, shows the following inequality:

$$
\frac{\left(x_{1}-\bar{X}\right)^{2}+\left(x_{2}-\bar{X}\right)^{2}+\ldots+\left(x_{n}-\bar{X}\right)^{2}}{n} \leq \frac{1}{4}(Q-q)^{2},
$$


where $Q=\max \left\{x_{1}, x_{2}, \ldots, x_{n}\right\}$ and $q=\min \left\{x_{1}, x_{2}, \ldots, x_{n}\right\}$. This inequality suggests an upper bound for indicators: dispersion, standard deviation and coefficient of variation, thus:

$$
\operatorname{Var}(X) \leq \frac{1}{4}(Q-q)^{2}, \sigma_{\bar{X}} \leq \frac{1}{2}(Q-q) \text { and } C_{V} \leq \frac{Q-q}{2 \bar{X}}
$$

In 2000, Bhatia and Davis, show in [12] that the dispersion is less or equal than the product differences between maximum and mean value, respectively mean and minimum value, thus,

$$
\operatorname{Var}(X) \leq(Q-\bar{X})(\bar{X}-q)
$$

which is equivalent with the inequality:

$$
\frac{\left(x_{1}-\bar{X}\right)^{2}+\left(x_{2}-\bar{X}\right)^{2}+\ldots+\left(x_{n}-\bar{X}\right)^{2}}{n} \leq(Q-\bar{X})(\bar{X}-q) .
$$

Because the term $(Q-\bar{X})(\bar{X}-q)$ is less or equal than the term $\frac{1}{4}(Q-q)^{2}$, we obtain that the inequality (18) is better than inequality (15). Thus, we obtain upper bounds better than in the relation (16):

$$
\begin{aligned}
\operatorname{Var}(X) & \leq(Q-\bar{X})(\bar{X}-q) \\
\sigma_{\bar{X}} & \leq \sqrt{(Q-\bar{X})(\bar{X}-q)}, \\
C_{V} & \leq \frac{\sqrt{(Q-\bar{X})(\bar{X}-q)}}{\bar{X}} .
\end{aligned}
$$

It has been shown in [13] by Mercer that for a sample of real numbers,

$$
\operatorname{Var}(X) \leq 2 Q\left(\bar{X}-\bar{X}_{h}\right)
$$

where $\bar{X}_{h}$ is the harmonic mean of the sample, i.e., $\bar{X}_{h}=\frac{n}{\sum_{i=1}^{n} \frac{1}{x_{i}}}$.

Next, we will present several improvements of the above inequalities related to variance.

Theorem 5. For a sample of real number $X$ there is the following inequality:

$$
2 q\left(\bar{X}-\bar{X}_{g}\right) \leq \operatorname{Var}(X) \leq 2 Q\left(\bar{X}-\bar{X}_{g}\right) .
$$

Proof. In the paper [14], Cartwright and Field, proved the following inequality:

$$
\begin{aligned}
\frac{1}{2 Q} \sum_{i=1}^{n} \alpha_{i}\left(x_{i}-\sum_{i=1}^{n} \alpha_{i} x_{i}\right)^{2} & \leq \sum_{i=1}^{n} \alpha_{i} x_{i}-\prod_{i=1}^{n} x_{i}^{\alpha_{i}} \\
& \leq \frac{1}{2 q} \sum_{i=1}^{n} \alpha_{i}\left(x_{i}-\sum_{i=1}^{n} \alpha_{i} x_{i}\right)^{2}
\end{aligned}
$$

where $\alpha_{i}>0, \forall i=1, \ldots, n$ and $\sum_{i=1}^{n} \alpha_{i}=1$.

For $\alpha_{i}=\frac{1}{n}$, for all $i=1, \ldots, n$, in inequality (22), we will obtain:

$$
\begin{aligned}
& \frac{1}{2 Q} \cdot \frac{\left(x_{1}-\bar{X}\right)^{2}+\left(x_{2}-\bar{X}\right)^{2}+\ldots+\left(x_{n}-\bar{X}\right)^{2}}{n} \leq \\
& \bar{X}-\bar{X}_{g} \leq \frac{1}{2 q} \cdot \frac{\left(x_{1}-\bar{X}\right)^{2}+\left(x_{2}-\bar{X}\right)^{2}+\ldots+\left(x_{n}-\bar{X}\right)^{2}}{n},
\end{aligned}
$$

thus, $2 q\left(\bar{X}-\bar{X}_{g}\right) \leq \operatorname{Var}(X) \leq 2 Q\left(\bar{X}-\bar{X}_{g}\right)$. 
Remark 3. It is easy to see that inequality (21) is a refinement of inequality (20). Thus, we have situations when a bound is better then the other. This inequality provide another upper bound for the dispersion $\operatorname{Var}(X)$, which makes us to compare the terms $2 Q\left(\bar{X}-\bar{X}_{g}\right)$ and $(Q-\bar{X})(\bar{X}-q)$, to see which of them is less.

We consider, the real number $a>1$ and choose the values $x_{1}=a-1, x_{2}=a, x_{3}=a+1$, to compare the bounds $M_{1}=(Q-\bar{X})(\bar{X}-q)$ and $M_{2}=2 Q\left(\bar{X}-\bar{X}_{g}\right)$. For respective values we have $q=a-1$, $x=a, Q=a+1$, which means that $M_{1}=1$ and $M_{2}=2(a+1)\left(a-\sqrt[3]{a^{3}-a}\right)$. We consider the function $f:(0,+\infty) \rightarrow R, f(x)=2(x+1)\left(x-\sqrt[3]{x^{3}-x}\right)-1$

Studying this function, we found that exists $c=2,428 \ldots$, where $f^{\prime}(c)=0$, thus for $x \in(1, c)$, we have $M_{1}<M_{2}$ and for $x \in(c, \infty)$, we have $M_{1}>M_{2}$. When the arithmetical mean of three consecutive numbers is greater than $c$, we have $M_{1}>M_{2}$, and when the arithmetical mean of three consecutive numbers is between 1 and $c$, then $M_{1}<M_{2}$. Thus, we have situations when a bound is better then the other.

Combining the above inequalities and taking into account Remark 3, we found other bounds for dispersion $\operatorname{Var}(X)$, standard deviation and coefficient of variation:

$$
\begin{aligned}
& 2 q\left(\bar{X}-\bar{X}_{g}\right) \leq \operatorname{Var}(X) \leq \min \left\{2 Q\left(\bar{X}-\bar{X}_{g}\right),(Q-\bar{X})(\bar{X}-q)\right\} \\
& \sqrt{2 q\left(\bar{X}-\bar{X}_{g}\right)} \leq \sigma_{\bar{X}} \leq \sqrt{\min \left\{2 Q\left(\bar{X}-\bar{X}_{g}\right),(Q-\bar{X})(\bar{X}-q)\right\}}
\end{aligned}
$$

and

$$
\frac{\sqrt{2 q\left(\bar{X}-\bar{X}_{g}\right)}}{\bar{X}} \leq C_{V} \leq \min \left\{2 Q\left(\bar{X}-\bar{X}_{g}\right),(Q-\bar{X})(\bar{X}-q)\right\} .
$$

Now, we want to find an upper bound, better than the Bhatia and Davis, for the above indicators. For the beginning, we establish:

Theorem 6. For a sample of real number $X$ there is the following equality:

$$
(Q-\bar{X})(\bar{X}-q)-\operatorname{Var}(X)=\frac{1}{n} \sum_{i=1}^{n}\left(Q-x_{i}\right)\left(x_{i}-q\right)
$$

Proof. We observe that:

$$
\begin{aligned}
& \frac{1}{n} \sum_{i=1}^{n}\left(Q-x_{i}\right)\left(x_{i}-q\right)=\frac{1}{n}\left[(Q+q) \sum_{i=1}^{n} x_{i}-\sum_{i=1}^{n} x_{i}^{2}-Q q\right] \\
& =(Q+q) \bar{X}-\frac{1}{n} \sum_{i=1}^{n} x_{i}^{2}-Q q \\
& =(Q+q) \bar{X}-\operatorname{Var}(X)-\bar{X}^{2}-Q q=(Q-\bar{X})(\bar{X}-q)-\operatorname{Var}(X)
\end{aligned}
$$

Theorem 7. For a sample of real number $X$ if $Q=\max \left\{x_{1}, x_{2}, \ldots, x_{n}\right\}, q=\min \left\{x_{1}, x_{2}, \ldots, x_{n}\right\}, Q^{\prime}=$ $\max \left\{x_{i} \mid x_{i} \neq Q\right\}$ and $q^{\prime}=\min \left\{x_{i} \mid x_{i} \neq q\right\}$, then there is the following inequality:

$$
\operatorname{Var}(X) \leq(Q-\bar{X})(\bar{X}-q)-\max \left\{\left(q^{\prime}-q\right)(Q-\bar{X}),\left(Q-Q^{\prime}\right)(\bar{X}-q)\right\}
$$

Proof. for $Q=\max \left\{x_{1}, x_{2}, \ldots, x_{n}\right\}$ and $q=\min \left\{x_{1}, x_{2}, \ldots, x_{n}\right\}$, we have: $Q \geq x_{i}$, and $q \leq x_{i}$, thus, $\left(Q-x_{i}\right)\left(x_{i}-q\right) \geq 0$.

Consequently, the proof of inequality (17) is obvious.

For $Q^{\prime}=\max \left\{x_{i} \mid x_{i} \neq Q\right\}$ and $q^{\prime}=\min \left\{x_{i} \mid x_{i} \neq q\right\}$, if we replace the term $Q-x_{i}$ with $Q-Q^{\prime}$ in relation (26), we obtain:

$$
(Q-\bar{X})(\bar{X}-q)-\operatorname{Var}(X) \geq \frac{1}{n} \sum_{i=1}^{n}\left(Q-Q^{\prime}\right)\left(x_{i}-q\right)=\left(Q-Q^{\prime}\right)(\bar{X}-q)
$$


and if we replace $x_{i}-q$ with $q^{\prime}-q$, we have:

$$
(Q-\bar{X})(\bar{X}-q)-\operatorname{Var}(X) \geq\left(q^{\prime}-q\right)(Q-\bar{X}),
$$

which means that inequality (17) has been improved by the inequality of the statement.

Remark 4. We obtain the next bounds for variance, standard deviation and coefficient of variation:

$$
\begin{aligned}
2 q\left(\bar{X}-\bar{X}_{g}\right) \leq & \operatorname{Var}(X) \\
& \leq(Q-\bar{X})(\bar{X}-q)- \\
& -\max \left\{\left(q^{\prime}-q\right)(Q-\bar{X}),\left(Q-Q^{\prime}\right)(\bar{X}-q)\right\}, \\
\sqrt{2 q\left(\bar{X}-\bar{X}_{g}\right)} \leq & \sigma_{\bar{X}} \\
\leq & \sqrt{(Q-\bar{X})(\bar{X}-q)-\max \left\{\begin{array}{c}
\left(q^{\prime}-q\right)(Q-\bar{X}), \\
\left(Q-Q^{\prime}\right)(\bar{X}-q)
\end{array}\right\},}
\end{aligned}
$$

and

$$
\begin{aligned}
\frac{\sqrt{2 q\left(\bar{X}-\bar{X}_{g}\right)}}{\bar{X}} & \leq C_{V} \\
& \leq \frac{\sqrt{(Q-\bar{X})(\bar{X}-q)-\max \left\{\left(q^{\prime}-q\right)(Q-\bar{X}),\left(Q-Q^{\prime}\right)(\bar{X}-q)\right\}}}{\bar{X}} .
\end{aligned}
$$

For a discrete random variableX $=\left(\begin{array}{c}x_{i} \\ p_{i}\end{array}\right)_{1 \leq i \leq n}$, we take $Q \geq \max \left\{x_{1}, x_{2}, \ldots, x_{n}\right\}$ and $q \leq$ $\min \left\{x_{1}, x_{2}, \ldots, x_{n}\right\}$. we have $x_{i} \neq x_{j}, \forall i \neq j, i, j=\overline{1, n}$.

We consider the following discrete random variables: $Q-X=\left(\begin{array}{c}Q-x_{i} \\ \frac{1}{n}\end{array}\right)_{1 \leq i \leq n}$, and $X-q=$ $\left(\begin{array}{c}x_{i}-q \\ \frac{1}{n}\end{array}\right)_{1 \leq i \leq n}$

It is easy to see that $\operatorname{Var}(Q-X)=\operatorname{Var} X$ and $\operatorname{Var}(X-q)=\operatorname{Var} X$, if $a_{i}=Q-x_{i}$ and $b_{i}=x_{i}-q$, then we obtain:

$$
\sum_{i=1}^{n} a_{i} b_{i}=n((Q-\bar{X})(\bar{X}-q)-\operatorname{Var}(X)) .
$$

Next, we calculate $\sum_{i=1}^{n} a_{i}^{2}$ and $\sum_{i=1}^{n} b_{i}^{2}$, for $a_{i}$ and $b_{i}$ given above. Thus, we deduce:

$$
\begin{aligned}
& \sum_{i=1}^{n} a_{i}^{2}=\sum_{i=1}^{n}\left(Q-x_{i}\right)^{2}=n\left(\operatorname{Var}(Q-X)+(Q-\bar{X})^{2}\right) \\
& =n\left(\operatorname{Var}(X)+(Q-\bar{X})^{2}\right)
\end{aligned}
$$

and

$$
\begin{aligned}
& \sum_{i=1}^{n} b_{i}^{2}=\sum_{i=1}^{n}\left(x_{i}-q\right)^{2}=n\left(\operatorname{Var}(X-q)+(\bar{X}-q)^{2}\right) \\
& =n\left(\operatorname{Var}(X)+(\bar{X}-q)^{2}\right) .
\end{aligned}
$$

Therefore, we have:

$$
\begin{aligned}
& \sum_{i=1}^{n} a_{i}^{2} \cdot \sum_{i=1}^{n} b_{i}^{2}-\left(\sum_{i=1}^{n} a_{i} b_{i}\right)^{2} \\
& =n^{2}\left(\left(\operatorname{Var}(X)+(Q-\bar{X})^{2}\right)\left(\operatorname{Var}(X)+(\bar{X}-q)^{2}\right)\right. \\
& \left.-((Q-\bar{X})(\bar{X}-q)-\operatorname{Var}(X))^{2}\right) \\
& =n^{2} \operatorname{Var}(X) \cdot(Q-q)^{2} .
\end{aligned}
$$


However, the second term of inequality (2) becomes

$$
\begin{gathered}
\max _{i, j \in\{1, \ldots, n\}} \frac{\left(a_{i} b_{j}-a_{j} b_{i}\right)^{2}}{b_{i}^{2}+b_{j}^{2}} \sum_{i=1}^{n} b_{i}^{2} \\
=n \cdot(Q-q)^{2} \max _{i, j \in\{1, \ldots, n\}} \frac{\left(x_{i}-x_{j}\right)^{2}}{\left(x_{i}-q\right)^{2}+\left(x_{j}-q\right)^{2}} \cdot\left(\operatorname{Var}(X)+(\bar{X}-q)^{2}\right), n \geq 2 .
\end{gathered}
$$

Thus, we obtain:

$$
n \cdot \operatorname{Var}(X) \geq\left(\operatorname{Var}(X)+(\bar{X}-q)^{2}\right) \max _{i, j \in\{1, \ldots, n\}} \frac{\left(x_{i}-x_{j}\right)^{2}}{\left(x_{i}-q\right)^{2}+\left(x_{j}-q\right)^{2}} .
$$

For $n \geq 2$, taking into account that $\operatorname{Var}(X) \geq 0$, we proved that inequality:

$$
\operatorname{Var}(X) \geq \frac{(\bar{X}-q)^{2}}{n} \max _{i, j \in\{1, \ldots, n\}} \frac{\left(x_{i}-x_{j}\right)^{2}}{\left(x_{i}-q\right)^{2}+\left(x_{j}-q\right)^{2}} .
$$

Similarly, we deduce:

$$
\operatorname{Var}(X) \geq \frac{(Q-\bar{X})^{2}}{n} \max _{i, j \in\{1, \ldots, n\}} \frac{\left(x_{i}-x_{j}\right)^{2}}{\left(Q-x_{i}\right)^{2}+\left(Q-x_{j}\right)^{2}} .
$$

We study, two cases:

(I) for $A=B=\sqrt{n\left(\operatorname{Var}(X)+(Q-q)^{2}\right)}$, we have:

$$
\begin{gathered}
A B-\sum_{i=1}^{n} a_{i} b_{i}=n\left(2 \operatorname{Var}(X)+(Q-q)^{2}-(Q-\bar{X})(\bar{X}-q)\right)>0, \\
A^{2}-\sum_{i=1}^{n} a_{i}^{2}=n(\bar{X}-q)(2 Q-q-\bar{X})>0, \text { for every } n \geq 2, \\
B^{2}-\sum_{i=1}^{n} b_{i}^{2}=n(Q-\bar{X})(Q+\bar{X}-2 q)>0, \text { for every } n \geq 2 .
\end{gathered}
$$

Therefore, applying the Aczél inequality, we proved:

$$
\begin{aligned}
& \left(2 \operatorname{Var}(X)+(Q-q)^{2}-(Q-\bar{X})(\bar{X}-q)\right)^{2} \\
& \geq(Q-\bar{X})(\bar{X}-q)(Q+\bar{X}-2 q)(2 Q-q-\bar{X}),
\end{aligned}
$$

which means that:

$$
\begin{aligned}
& \operatorname{Var}(X) \geq \frac{1}{2}(\sqrt{(Q-\bar{X})(\bar{X}-q)(Q+\bar{X}-2 q)(2 Q-q-\bar{X})} \\
& \left.+(Q-\bar{X})(\bar{X}-q)-(Q-q)^{2}\right) .
\end{aligned}
$$

But, using the improvement of Aczél inequality, from inequality (12), we obtain:

$$
\begin{aligned}
& \operatorname{Var}(X) \geq \frac{1}{2}(\sqrt{(Q-\bar{X})(\bar{X}-q)(Q+\bar{X}-2 q)(2 Q-q-\bar{X})} \\
& \left.+(Q-\bar{X})(\bar{X}-q)-(Q-q)^{2}\right)+P
\end{aligned}
$$

where

$$
P=\frac{1}{2 n} \max _{i \in\{1, \ldots, n\}}\left\{A B-a_{i} b_{i}-\left(A^{2}-a_{i}^{2}\right)^{1 / 2}\left(B^{2}-b_{i}^{2}\right)^{1 / 2}\right\},
$$


and $A=B=\sqrt{n\left(\operatorname{Var}(X)+(Q-q)^{2}\right)}$.

(II) For

$$
\begin{aligned}
& A=\sqrt{n\left((Q-\bar{X})(\bar{X}-q)+(Q-\bar{X})^{2}\right)} \\
& =\sqrt{n(Q-\bar{X})(Q-q)}
\end{aligned}
$$

and

$$
\begin{aligned}
& B=\sqrt{n\left((Q-\bar{X})(\bar{X}-q)+(\bar{X}-q)^{2}\right)} \\
& =\sqrt{n(\bar{X}-q)(Q-q)}
\end{aligned}
$$

we deduce:

$$
\begin{gathered}
A B-\sum_{i=1}^{n} a_{i} b_{i}=n(Q-q) \sqrt{(Q-\bar{X})(\bar{X}-q)} \\
-(Q-\bar{X})(\bar{X}-q)+\operatorname{Var}(X)>0 . \\
A^{2}-\sum_{i=1}^{n} a_{i}^{2}=n\left((Q-\bar{X})(Q-q)-\operatorname{Var}(X)-(Q-\bar{X})^{2}\right) \\
=n((Q-\bar{X})(\bar{X}-q)-\operatorname{Var}(X))>0
\end{gathered}
$$

and

$$
\begin{gathered}
B^{2}-\sum_{i=1}^{n} b_{i}^{2}=n\left((\bar{X}-q)(Q-q)-\operatorname{Var}(X)-(\bar{X}-q)^{2}\right) \\
=n((Q-\bar{X})(\bar{X}-q)-\operatorname{Var}(X))>0 .
\end{gathered}
$$

Consequently, if we use the Aczél inequality, we find:

$$
\begin{gathered}
((Q-q) \sqrt{(Q-\bar{X})(\bar{X}-q)}-(Q-\bar{X})(\bar{X}-q)+\operatorname{Var}(X))^{2} \\
\geq((Q-\bar{X})(\bar{X}-q)-\operatorname{Var}(X))^{2} \\
\operatorname{Var}(X) \geq(Q-\bar{X})(\bar{X}-q)-\frac{Q-q}{2} \sqrt{(Q-\bar{X})(\bar{X}-q)} .
\end{gathered}
$$

But, using the Aczél inequality refinement, from inequality (12), we find:

$$
\operatorname{Var}(X) \geq(Q-\bar{X})(\bar{X}-q)-\frac{Q-q}{2} \sqrt{(Q-\bar{X})(\bar{X}-q)}+P^{\prime},
$$

where

$$
P^{\prime}=\frac{1}{2 n} \max _{i \in\{1, \ldots, n\}}\left\{A B-a_{i} b_{i}-\left(A^{2}-a_{i}^{2}\right)^{1 / 2}\left(B^{2}-b_{i}^{2}\right)^{1 / 2}\right\},
$$

and $A=\sqrt{n(Q-\bar{X})(Q-q)}$ and $B=\sqrt{n(\bar{X}-q)(Q-q)}$.

\section{Conclusions}

Based on the results obtained in this paper regarding the new refinements for the main indicators characterizing the scattering, we will try in the future to find other applications of Aczél inequality respectively Popoviciu's inequality to other statistical indicators. For example, we will extend the inequality from Theorem 4 to $\mathrm{m}$ sequences of positive real numbers. In the future we will look for other applications demonstrated in the article and in other fields. we will also study what variants from these results can be extended in the inner product spaces. 
Author Contributions: The work presented here was carried out in collaboration between both authors. Both authors contributed equally and significantly in writing this article. Both authors have contributed to the manuscript. Both authors have read and agreed to the published version of the manuscript.

Funding: Project financed by Lucian Blaga University of Sibiu \& Hasso Plattner Foundation research grants LBUS-IRG-2019-05.

Acknowledgments: The authors would like to thank the reviewers and the editors for their useful suggestions.

Conflicts of Interest: The authors declare no conflict of interest. The funders had no role in the design of the study; in the collection, analyses, or interpretation of data; in the writing of the manuscript, or in the decision to publish the results.

\section{References}

1. Dragomir, S.S.; Mond, B. Some inequalities of Aczél type for Gramians in inner product spaces. Nonlinear Funct. Anal. Appl. 2001, 6, 411-424.

2. Mascioni, V. A note on Aczél type inequalities. J. Inequal. Pure Appl. Math. 2002, 3, 69.

3. Wu, S. Some improvements of Aczél's s inequality and Popoviciu's inequality. Comput. Math. Appl. 2008, 56, $1196-1205$. [CrossRef]

4. Mărghidanu, D.; Díaz-Barrero, J.L.; Rădulescu, S. New refinements of some classical inequalities. Math. Inequal. Appl. 2009, 12, 513-518. [CrossRef]

5. Pop, O. About Bergström inequality. J. Math. Ineq. 2009, 3, 237-242. [CrossRef]

6. Aczél, J. Some general methods in the theory of functional equations in one variable. New applications of functional equations (Russian). Uspehi Mat. Nauk (N.S.) 1956, 11, 3-68.

7. Popoviciu, T. On an inequality. Gazeta Matematica si Fizica. Seria A (Romanian) 1959, 11, 451-461.

8. Mărghidanu, D. Generalizations and refinements for Bergström and Radon's inequalities. J. Sci. Arts 2008, 8, 57-62.

9. Raţiu, A.; Minculete, N. Several refinements and counterparts of Radon's inequality. Math. Bohem. 2015, 140, 71-80.

10. Qi, F.; Cerone, P.; Dragomir, S; Srivastava, H.M. Alternative proofs for monotonic and logarithmically convex properties of one-parameter mean values. Appl. Math. Comput. 2009, 208, 129-133. [CrossRef]

11. Evans, J.R. Statistics. Data Analysis and Decision Modeling; Pearson Prentice Hall: Upper Saddle River, NJ, USA, 2007.

12. Bhatia, R.; Davis, C. A better bound on the variance. Am. Math. Mon. 2000, 107, 353-357. [CrossRef]

13. Mercer, A.M. Bounds for A-G, A-H, G-H, and a family of inequalities of Ky Fan's type, using a general method. J. Math. Anal. Appl. 2000, 243, 163-173. [CrossRef]

14. Cartwright, D.I.; Field M.J. A refinement of the arithmetic mean-geometric mean inequality. Proc. Am. Math. Soc. 1978, 71, 36-38. [CrossRef]

(C) 2020 by the authors. Licensee MDPI, Basel, Switzerland. This article is an open access article distributed under the terms and conditions of the Creative Commons Attribution (CC BY) license (http:/ / creativecommons.org/licenses/by/4.0/). 